Revue d'histoire de l'Amérique française

\#A REVUE D.HISTOIRE DE L'AMÉRIQUE FRANÇAISE

\title{
Établissement du séminaire royal pour les missions d'Amérique
}

\section{Honorius Provost}

Volume 9, numéro 4, mars 1956

URI : https://id.erudit.org/iderudit/301788ar

DOI : https://doi.org/10.7202/301788ar

Aller au sommaire du numéro

Éditeur(s)

Institut d'histoire de l'Amérique française

\section{ISSN}

0035-2357 (imprimé)

1492-1383 (numérique)

Découvrir la revue

\section{Citer cet article}

Provost, H. (1956). Établissement du séminaire royal pour les missions d'Amérique. Revue d'histoire de l'Amérique française, 9(4), 502-511. https://doi.org/10.7202/301788ar d'utilisation que vous pouvez consulter en ligne.

https://apropos.erudit.org/fr/usagers/politique-dutilisation/ 


\section{Documents}

\section{«ÉTABLISSEMENT DU SÉMINAIRE ROYAL POUR LES MISSIONS D'AMÉRIQUE »}

L'épopée mystique de la Nouvelle-France, comme on l'a justement désignée, a pris origine dans la première moitié du $17^{\mathrm{ème}}$ siècle, alors que la vie religieuse en France connaissait ellemême une féconde période de mysticisme. La piété et l'esprit d'apostolat habitaient couramment à l'enseigne du grand monde et jusqu'aux palais des princes.

On ne saurait trop rappeler à notre élite catholique d'aujourd'hui, que toutes nos premières fondations religieuses ont pris naissance - peut-être parce qu'elles ne l'auraient pu autrement, grâce à la libéralité et à la piété d'éminents citoyens de la mèrepatrie, laïcs plus encore qu'ecclésiastiques. L'énumération des plus connus est assez imposante: l'abbé Charles des Boves, pour les Récollets; Noël Brûlart de Sillery et le Marquis de Gamaches, pour les Jésuites; Mme de la Peltrie, pour les Ursulines; la duchesse d'Aiguillon, cette légendaire nièce du Cardinal de Richelieu, pour l'Hôtel-Dieu de Québec et, plus tard, pour le Séminaire des Missions Étrangères; le baron de Fancamp, pour la Société Notre-Dame de Montréal; Mme de Bullion, pour l'Hôtel-Dieu du même endroit. Autre fait notable: plusieurs de ces éminents laïcs menaient dans le monde la vie religieuse authentique, par exemple Mme de la Peltrie, Jeanne Mance; on l'a dit de Maisonneuve et même de Montmagny. D'autres finissaient par s'inscrire à l'état ecclésiastique, en particulier MM. de Sillery et Charles de Lauzon, le fils du gouverneur, gouverneur lui-même, par interim.

Ce fut le cas pareillement d'un personnage connu à peu près de nom seulement dans notre histoire et qui mérite à coup sûr plus d'égards; nous voulons parler du quatrième vice-roi de 
la Nouvelle-France; Henry de Lévis duc de Ventadour. C'est à lui que la ville de Lévis doit son appellation, malheureusement déformée au cours des siècles.

On possède quelques renseignements sur la vie de ce personnage de haute noblesse. Né en 1596, il était le fils d'Anne de Lévis, duc de Ventadour et de Marguerite de Montmorency. Voici la notice que donne de lui un ancien recueil, publié à Paris en 1728: ${ }^{1}$ « Henry de Lévis, duc de Ventadour, Pair de France, ${ }^{2}$ prince de Maubuisson, lieutenant general de Languedoc, comte de la Voute, Tournon \&c. Il avoit été reçu au parlement en qualité de duc de Ventadour, Pair de France le 21. decembre 1624. Dans la suite, désirant se faire ecclesiastique, il ceda sa dignité de duc de Ventadour au marquis d'Annonay son frere, par acte du 23. may 1631. fut chanoine de l'église de Notre-Dame de Paris, directeur general des Seminaires, s'étant auparavant séparé de sa femme; il mourut le 14. octobre 1680. âgé de 84 . ans, $\&$ fut enterré en l'église de Notre-Dame. - Femme, Marie-Liesse de Luxembourg, princesse de Tingry, fille d'Henry, duc de Luxembourg. \& de Madelene de Montmorency, dame de Thoré: se fit du consentement de son mary, religieuse Carmelite dans le couvent de Chambery, qu'elle fonda ».

On trouve plus de détails sur ce ménage extraordinaire, dans un ouvrage anonyme publié à Paris, en 1889, et intitulé: Le duc et la duchesse de Ventadour; un grand amour chrétien au dix-septième siècle. La duchesse avait été élevée par la mère de son futur époux. A leur mariage, en 1623, il avait 27 ans; elle en avait 12. Ils continuèrent à vivre en se considérant comme frère et sœur. Elle rêvait graduellement du cloître; lui rêvait de la vie de prêtre. Avec l'autorisation du Pape, ils se séparèrent au carmel d'Avignon, le 19 septembre 1629. La nouvelle moniale y prononça ses vœux en 1634 et alla fonder peu après le couvent de Chamberry, où elle mourut le 18 janvier 1660. Son mari attendit en 1641 pour entrer dans les ordres sacrés.

1 Histoire généalogique et chronologique de la Maison royale de France, des pairs, grands officiers de la Couronne... par le P. Anselme... Tome IV : $31 \mathrm{~s}$.

2 Un des premiers dignitaires du royaume, grand vassal de la couronne et faisant partie du Conseil du Roi. 
Mais ses aspirations apostoliques l'avaient poussé dès avant ce temps à tenter des projets grandioses. Il avait de la fortune et trouvait légitime de l'accroître, soit par la force des armes, soit par l'acquisition de bénéfices. Mais il brûlait de mettre son nom et ses richesses au service des bonnes œuvres.

Rochemonteix nous raconte, dans Les Jésuites de la Nouvelle-France (volume I), comment il devint vice-roi de la Nouvelle-France. Son oncle, Henri II, duc de Montmorency, détenait cette fonction, qu'il avait achetée 11,000 écus $(38,000$ francs) de son beau-frère, le prince de Condé, «preuve certaine, dit F.-X. Garneau, que cette charge honorifique avait déjà de la valeur ». La compagnie de commerce versait au vice-roi une forte annuité. Mais le duc de Montmorency en avait assez des obstacles qu'on lui suscitait de tous côtés. Stimulé par les Jésuites, ses conseillers, le duc de Ventadour acheta donc la charge de son oncle, en janvier 1625, au prix énorme de 100,000 livres. Dès le printemps de la même année, il assurait à ses frais la venue à Québec des cinq premiers missionnaires Jésuites, trois Pères et deux Frères.

Ce changement de «vice-rois fainéants », comme les qualifie Emile Salone (La colonisation de la Nouvelle-France, 1906, p. 29), était à peu près sans importance pour le Canada, à ne considérer que le point de vue politique. Mais, cette fois, il marquait une avance formidable au point de vue spirituel. F.-X. Garneau dit de Ventadour: «En acquérant cette charge, il voulait travailler moins à l'établissement du Canada qu'à la conversion des infidèles. Aussi négligea-t-il entièrement la colonisation pour ne s'occuper que des missions ». Notons toutefois à sa décharge, et c'est un fait notoire, que les autres vicerois n'avaient pas fait mieux que lui pour la colonisation, en laissant les compagnies de commerce agir à leur guise, au grand mécontentement de Champlain, le lieutenant-général. Il faudra, pour changer ce régime, le coup de barre vigoureux de Richelieu, en 1628, par la fondation de la Compagnie des Cent-Associés. L'influence du duc de Ventadour en Nouvelle-France cessa donc bientôt après sa première manifestation. 
Cependant il ne tarda pas à lui substituer une autre entreprise apostolique. Au mois de mai 1627 , il eut soudain une inspiration plus ou moins vague, mais dont la beauté le ravit. Il y avait probablement beaucoup d'illuminisme dans son projet; il réussit quand même à le faire aboutir, grâce à l'assistance de compagnons sans doute plus avisés que lui, et, de ce jour, naquit la grande Compagnie du Saint-Sacrement, la «cabale des dévôts », comme on l'a dénommée, qui en viendrait à dominer la France, comme une puissance occulte, pendant un bon quart de siècle, avant d'être maîtrisée et dissoute par Louis XIV et Colbert. C'était une association secrète, comptant de multiples cellules à travers le royaume et des adhérents de tous noms et de toutes classes; c'était une cinquième colonne d'action catholique, élevée contre le jansénisme, l'irréligion et l'immoralité. Il y eut chez elle d'authentiques saints, comme Vincent de Paul, un des membres les plus actifs et les plus influents. Bossuet, Fénélon en firent partie, et d'autres qui nous intéressent de plus près: Jean de Bernières de Louvigny, François de MontmorencyLaval, M. de Maizerets, la duchesse d'Aiguillon, le baron de Fancamp, M. Olier et M. de la Dauversière - les deux derniers, qu'on prétend s'être rencontrés "par hasard», pour former la Société de Notre-Dame de Montréal; bien d'autres encore. Il y aurait long à écrire sur l'influence directe ou indirecte de la Compagnie du Saint-Sacrement dans les origines religieuses du Canada; les archives de la Compagnie ne sont pas encore toutes exploitées. $^{3}$

Quant au duc de Ventadour, après avoir été le père et le premier directeur du mouvement, il passa vite à l'arrière-plan. Il pouvait avoir bien des qualités; il lui manquait apparemment l'esprit pratique. Et saint Vincent de Paul pourra écrire, en faisant visiblement allusion à ce personnage: «J'ai connu des hommes saints professant l'épée, sans compter ceux des autres professions, qui y faisaient merveille et qui, étant passés en l'état ecclésiastique, n'y ont fait presque aucun bien ». ${ }^{4}$ Mais il

3 Raoul Allier, La Cabale des Dévots (Paris, 1902).

4 Lettres, II : 385. 
est temps d'en venir à l'objet de notre document: le Séminaire Royal pour les missions d'Amérique.

Le mot séminaire a aujourd'hui des acceptions dont on peut se demander quelle relation elles ont avec l'étymologie et avec l'acception traditionnelle. On a, en effet, dans les facultés universitaires, des séances de travaux pratiques pour les élèves, qu'on appelle séminaires ou seminars, suivant l'orthographe anglaise. On appelle souvent de la même façon certaines conventions où des délégués de divers organismes ou de divers pays se rencontrent pour discuter amicalement des problèmes d'actualité. Mais le sens premier de séminaire, c'est pour désigner un lieu où l'on prépare des sujets à remplir les fonctions de ministres du culte. Ainsi l'entendait déjà le Dictionnaire de Trévoux (édition 1752). L'institution canonique des séminaires remonte au Concile de Trente, qui «ordonne de prendre des enfans au-dessus de douze ans pour les nourrir en commun, et les instruire, et les rendre capables de parvenir à l'état Ecclésiastique ».

Cependant, outre ce sens strict, on avait jadis une acception plus large, s'appliquant à toute institution visant à donner l'instruction religieuse, même la plus élémentaire. Ainsi, on avait tout aussi bien des séminaires féminins. Le Dictionnaire de Trévoux donne comme exemple les Séminaires de l'Union Chrétienne, en France, où des veuves et des filles pieuses se consacraient à la conversion des femmes hérétiques et à l'instruction des nouvelles converties. Même chose se disait en pays d'infidélité. Combien de fois Marie de l'Incarnation ne parle-t-elle pas, dans ses écrits, du séminaire des Ursulines, pour la conversion des filles peaux-rouges de la colonie ? Et les Jésuites, en plus de leur Collège à Québec, eurent aussi un séminaire, à la résidence Notre-Dame des Anges, pour les indigènes payens ou néophytes.

Enfin, une autre signification du mot séminaire, cette fois plus apparentée à l'acception principale, désignait déjà les maisons ou associations groupant et préparant des clercs pour les 
missions en pays infidèles ou hérétiques. Le prototype de ces séminaires était bien celui de la Propagande, à Rome, et il l'est encore. Mais il a eu des répliques à maintes reprises et dans maints pays, en commençant par le Séminaire des Missions Etrangères de Paris, pour en venir aux multiples institutions analogues de notre époque.

C'est dans cette dernière catégorie, croyons-nous, qu'il faut placer le Séminaire Royal pour les missions d'Amérique, dont notre document ci-après fait mention, encore qu'on ne puisse l'assurer absolument parce qu'on n'en connaît pas l'histoire. Etait-ce encore une «illumination »du pieux duc de Ventadour ou une initiative de la Compagnie du Saint-Sacrement, qui devait bien, pour rester cachée, faire intervenir quelqu'un de ses membres, apparemment à titre personnel ? Jusqu'à présent, nous ne pouvons le dire, bien qu'il fût difficile de concevoir la réalisation de ce vaste plan, sans le concours de collaborateurs fiables dans tous les coins du royaume, et de qui, alors, sinon des compagnons du Saint-Sacrement?

Sur la mise en marche de l'entreprise, nous n'avons pour lors aucune information. Quoi qu'il en soit, l'affaire remonte à la surface en 1651, encore à l'état de projet, peut-être même un projet différent. Le 3 novembre, le duc de Ventadour demande à la S. C. de la Propagande l'autorisation d'ériger en France un séminaire pour le service des missions d'Amérique (Archives du Vatican, section de Propaganda Fide, Scritture antiche, vol. 260, ff. 141 à 152). Plus que cela, saint Vincent de Paul est informé que le projet comporte une nouvelle congrégation religieuse, sous le nom de Missionnaires des Indes, et il proteste contre le nom, qui ressemble trop à celui de son institut, les prêtres de la Mission. «Je savais, écrit-il, que l'on se remuait ici pour entreprendre cette œuvre, mais je ne savais pas qu'on en fit la poursuite à Rome, et encore moins que l'on eût espérance de la faire ériger en congrégation. J'ai appris [...] que M. de Ventadour sera le préfet général de ces missions et comme le patriarche de l'Amérique, qui aura tout pouvoir du Pape et sans la permission duquel personne n'y pourra aller faire les fonctions ecclésiastiques. Si cela était, il serait fort à craindre qu'il n'y arrivast 
désordre, et, déjà il y a un sujet de division tout formé $\gg .^{5}$ Un séminaire pour les missions d'Amérique, des missionnaires pour les Indes; y a-t-il confusion? Nullement. Il s'agit des Indes Occidentales françaises, c'est-à-dire de la Guyane et des îles d'Amérique. En cela, rien qui concerne précisément le Canada.

Toutefois, le projet, caressé apparemment par plusieurs compagnons du Saint-Sacrement, n'aboutit à aucun résultat pratique. Monsieur Vincent avait fini par lui consentir sa collaboration, mais en disant à ses subalternes, moins surnaturels et moins désintéressés: "Si cette œuvre est de Dieu, nous aurions grand tort de la traverser; et si elle ne l'est pas, Dieu la détruira, quand il lui plaira $\gg .{ }^{6}$ Une des nombreuses intuitions du saint.

Mettons un épilogue à cette histoire de projets avortés pour un séminaire des missions. La Providence, un jour, prépare un concours de circonstances imprévisibles et utilise une voie détournée, celle des congrégations des Jésuites et d'un petit cénacle de «bons amis» formé dans leur sein. Le Saint Siège devient soudainement sympathique et accorde les vicaires apostoliques demandés pour la Nouvelle-France et l'Extrême-Orient; il autorise, en 1658, l'établissement d'une société et d'un séminaire pour le maintien de ces missions lointaines. La Compagnie du Saint-Sacrement s'active de nouveau, dispense de larges ressources pécuniaires et nomme une commission de certains de ses membres, pour mettre au monde la fondation longtemps caressée, et ce sera le Séminaire des Missions Étrangères. Le duc de Ventadour, cette fois, n'aura pas son mot à dire; mais l'institution élira domicile rue du Bac, chez Mgr Bernard de SainteThérèse, ancien évêque de Babylone à la retraite, qui avait acquis naguère une belle propriété en vue d'en faire lui-même un séminaire pour ses missions de Perse. La fondation réalisée, il ne restera plus qu'à la faire couronner du sceau officiel, pour ainsi dire, celui des lettres patentes royales. Elles seront signées par Louis XIV, en juillet 1663, et le Roi ne se doutera pas qu'il

5 Pierre Coste, éditeur: Saint Vincent de Paul...I I- Correspondance, IV: 292 ss.; lettre du 21 décembre 1651.

6 Ibidem, 363. 
exauce l'ultime désir de ceux que sa police poursuit à ce moment même. Car la Compagnie du Saint-Sacrement, désormais démasquée, est traquée, proscrite du royaume et déjà en train de se démembrer. Le Séminaire des Missions Étrangères, "c'a été le Benjamin qu'elle a enfanté au lit de la mort $\gg^{7}$

Honorius PRovost, ptre, Sous-archiviste, Séminaire de Québec.

\section{Établissement du Séminaire Royal ${ }^{8}$ pour les missions d'Amérique}

\section{BREVET DU ROY}

Aujourd'huy dernier jour de mars mil six cens quarente six, le Roy estant à Paris, voulant avancer l'Establissement du Seminaire Royal des missions ordonné pour la conversion des sauvages des Isles et terres fermes nouvellement descouvertes en l'Amérique. Sa Majesté ${ }^{9}$ de l'advis de la Reyne Regente Sa Mere, ${ }^{10}$ a accordé au Sieur de Vantadour Ecclesiastique, la permission de lever et percevoir, le denier à Dieu, ${ }^{11}$ qui se donne volontairement en toutes les foires, marchez, contracts, ventes, Eschanges, baux et autres actes et conventions, ou le dit denier à Dieu se donne, pour estre les dits deniers employez et appliquez au dit Establissement, que pour cet effect, touttes lettres necessaires seront adressées aux Sieurs Archevesques, Evesques et autres Ecclesiastiques de ce Royaume pour exhorter et admonester tous les peuples de leurs dioceses et Eglises, de remettre le dit denier à Dieu ès mains de ceux qui seront pour cet effect commis par le dit Sieur de Vantadour. M'ayant sa dite Majesté commandé de luy en expedier toutes lettres necessaires, Et

7 R. Allier, opus citatum, 158.

8 Archives Nationales, Collection Moreau de Saint-Méry, Série F, vol. I, f. 224. Copie aux Archives Publiques d'Ottawa, Série F⿳, volume 3, fol. $163-167$.

${ }^{9}$ Louis XIV, alors âgé de huit ans.

10 Anne d'Autriche.

11 Denier à Dieu; voici le sens qu'en donne le Dictionnaire de Trévoux, sans en préciser l'histoire: "C'est un arrhe, une pièce d'argent, une petite somme que donne, quand un marché est conclu, celui qui achète, ou qui loue quelque chose; au vendeur ou au locateur. Quelques-uns disent qu'on appelle cet arrhe denier à Dieu, parce qu'on le donne principalement pour en faire aumóne aux pauvres. Peut-être est-ce parce qu'on le donne en disant adieu, en se séparant, lorsque le marché est conclu $\gg$. 
cependant le present brevet qu'elle a voulu signer de sa main, et fait contresigner par moy son conseiller et Secretaire de ses commandemens.

\author{
Signé: Louis et de Lomenie
}

\title{
SENTENCE DU CHASTELET 12
}

A tous ceux qui ces presentes lettres verront. Louys Seguier chevalier baron de St Brisson, Seigneur des Ruaux et de St Firmin, conseiller du Roy, gentilhomme ordinaire de sa chambre, et garde de la prevosté de Paris, SALUT, sçavoir faisons que veu le brevet du Roy, donné a Paris le dernier jour de mars 1646, Signé Louis, et plus bas de Lomenie, par lequel appert, Sa Majesté voulant avancer l'establissement du Seminaire Royal des Missions, ordonnées pour la conversion des Sauvages des Isles et Terres fermes, nouvellement descouvertes en l'Amerique, auroit de l'advis de la Reyne Regente sa Mere, accordé à Messire Henry de Levy de Vantadour Ecclesiastique, la permission de lever et persevoir le denier à Dieu qui se donne volontairement en toutes foires, marchez, contracts, ventes, Eschanges, baux et autres actes et conventions ou le dit denier à Dieu se donne, pour estre les dits deniers employez et appliquez au dit Establissement, et que pour cet effect toutes lettres seront adressées aux Sieurs Archevesques et Evesques et autres Ecclesiastiques de ce Royaume, pour exhorter et admonester les peuples de leurs diocèses et Eglises, de remettre le dit denier à Dieu ès mains de ceux qui seront pour cet effect commis par le dit Sieur de Vantadour et la requeste à nous presentée par le dit Sieur de Vantadour, Directeur general des Seminaires, ${ }^{13}$ ordonnez par sa dite Majesté pour la conversion des dits Infidelles Establis en la chapelle de Nostre Dame de Lorette près Montmartre, tendante à ce qu'il nous pleust le faire jouir du contenu au dit brevet; attendu le consentem ${ }^{t}$ de plusieurs corps de marchands ${ }^{14}$ de cette ville de Paris, laquelle requeste veües les pièces

12 Le Châtelet, forteresse contemporaine de la Bastille, était le siège de la Prévôté de Paris, le tribunal de la justice ordinaire de la ville.

${ }_{13}$ Le duc de Ventadour ne pouvait se prévaloir de ce titre qu'en vertu d'une commission royale. Encore un titre plutôt honorifique dont les attributions sont difficiles à préciser, faute de documents.

${ }_{14}$ Le Dictionnaire de Trévoux nous renseigne sur «les six corps des marchands à Paris; ce sont les Merciers, les Fourreurs, les Epiciers, les Drapiers, les Bonnetiers et les Orfèvres. Les Marchands de vin prétendoient faire le septième corps $\gg$. Cependant, le rédacteur de la sentence du Châtelet ne leur concède pas cette faveur, puisqu'ils sont énumérés en second 
et brevet cy dessus esnoncez, auroit esté de nostre ordonnance communiqué au Procureur du Roy qui auroit declaré n'empescher l'execution du dit brevet selon sa forme et teneur, vu aussy le consentem $^{t}$ signé des Maistres et gardes de la marchandise de vin, jurez tailleurs, vinaigriers, patenostriers, corroyeurs, charcutiers, mareschaux, cordonniers, aiguilliers, faiseurs d'instrumens de musique, bourliers, jurez et gardes de la bonne teinture, coffretiers, passementiers, boutonniers, rotisseurs, boulangers, chappeliers, patissiers, tondeurs, layetiers, jurez et gardes de la marchandise de lingerie, menuisiers sculpteurs, peintres, pannachers, couvreurs de maisons et autres en datte des quinziesme et vingt uniesme janvier dernier, par lesquelles nous est apparu les susdits maîtres gardes et jurez ${ }^{15}$ avoir consenty, que les trois parts au total les deux seront mises ès mains du dit Seigneur de Vantadour, pour estre employez aux fins portées par le brevet de Sa Majesté, se reservant les dits maistres et gardes et jurez, l'autre tiers, pour estre par eux emploié aux necessitez des veufves, orphelins et autres pauvres honteux de leurs dits ars et mestiers, et tout veu et consideré; nous avons du consentement du procureur du Roy, auquel le tout a esté communiqué, ordonné que les deux tiers des deniers à Dieu qui seront volontairement donnez en toutes foires, marchez, contracts, ventes, Eschanges, baux et autres actes et conventions ou le dit denier à Dieu sera baillé, seront mis ès mains du dit Seigneur de Vantadour, ou en celle de celuy qui sera par luy nommé, pour en faire la recepte, pour estre les deniers procedans du dit denier à Dieu employés suivant et au desir du brevet de Sa dite Majesté, en tesmoins de ce nous avons fait sceller ces presentes; ce fut fait et donné par Messire Dreux D'Aubray chevalier Seigneur d'Offemont, Villiers et autres lieux, Conseiller du Roy en ses conseils d'Estat et privés, Lieutenant civil de la ville et vicomte de Paris, ce vendredy, dix neufie. jour de Juin 1648. Signé: Hubert avec paraphe ./.

lieu, en tête des corps des artisans. Des études savantes existent touchant la nature, le fonctionnement et l'importance des corporations dans la vie sociale, ainsi que les rivalités entre elles. Notre texte ici en suggère une idée.

15 Les maîtres, par opposition aux apprentis, étaient ceux qui avaient droit d'avoir boutique à leur compte. Les jurés étaient élus par ceux de leur corps pour avoir droit de visite sur les autres et faire observer les règlements de la communauté ou corporation. Enfin, les gardes, par rapport aux corps de marchands, avaient le rôle correspondant aux jurés, pour les artisans. Mais nous voyons ici les deux termes accolés l'un à l'autre. 